\title{
ON THE EXISTENCE OF $(k, l)$-KERNELS IN INFINITE DIGRAPHS: A SURVEY
}

\author{
H. Galeana-SÁnchez \\ AND \\ C. Hernández-Cruz \\ Instituto de Matemáticas \\ Universidad Nacional Autónoma de México \\ Ciudad Universitaria, México, D.F., C.P. 04510, México \\ e-mail: hgaleana@matem.unam.mx \\ cesar@matem.unam.mx
}

\begin{abstract}
Let $D$ be a digraph, $V(D)$ and $A(D)$ will denote the sets of vertices and arcs of $D$, respectively.

A $(k, l)$-kernel $N$ of $D$ is a $k$-independent (if $u, v \in N, u \neq v$, then $d(u, v), d(v, u) \geq k$ ) and $l$-absorbent (if $u \in V(D)-N$ then there exists $v \in N$ such that $d(u, v) \leq l)$ set of vertices. A $k$-kernel is a $(k, k-1)$-kernel.

This work is a survey of results proving sufficient conditions for the existence of $(k, l)$-kernels in infinite digraphs. Despite all the previous work in this direction was done for $(2,1)$-kernels, we present many original results concerning $(k, l)$-kernels for distinct values of $k$ and $l$.

The original results are sufficient conditions for the existence of $(k, l)$ kernels in diverse families of infinite digraphs. Among the families that we study are: transitive digraphs, quasi-transitive digraphs, right/left pretransitive digraphs, cyclically $k$-partite digraphs, $\kappa$-strong digraphs, $k$-transitive digraphs, $k$-quasi-transitive digraphs.
\end{abstract}

Keywords: kernel, $k$-kernel, $(k, l)$-kernel, infinite digraph.

2010 Mathematics Subject Classification: 05C20, 05C63.

\section{REFERENCES}

[1] J. Bang-Jensen and G. Gutin, Digraphs. Theory, Algorithms and Applications (Springer-Verlag, Berlin Heidelberg New York, 2002). 
[2] J. Bang-Jensen and J. Huang, Quasi-transitive digraphs, J. Graph Theory 20 (1995) 141-161. doi:10.1002/jgt.3190200205

[3] C. Berge, Graphs (North-Holland, Amsterdam New York, 1985).

[4] D. Bród, A. Włoch and I. Włoch, On the existence of $(k, k-1)$-kernels in directed graphs, J. Math. Appl. 28 (2006) 7-12.

[5] M. Chudnovsky, N. Robertson, P. Seymour and R. Thomas, The Strong Perfect Graph Theorem, Ann. of Math. 164 (2006) 51-229. doi:10.4007/annals.2006.164.51

[6] V. Chvátal, On the computational complexity of finding a kernel, Report No. CRM-300, Centre de Recherches Mathematiques, Universite de Montreal, 1973.

[7] V. Chvátal and L. Lovász, Every directed graph has a semi-kernel, Lecture Notes in Math. 411 (1974) 175. doi:10.1007/BFb0066192

[8] R. Diestel, Graph Theory 3rd Edition (Springer-Verlag, Berlin Heidelberg New York, 2005).

[9] P. Duchet, Graphes noyau-parfaits, Ann. Discrete Math. 9 (1980) 93-101. doi:10.1016/S0167-5060(08)70041-4

[10] P. Duchet and H. Meyniel, Kernels in directed graphs: a poison game, Discrete Math. 115 (1993) 273-276. doi:10.1016/0012-365X(93)90496-G

[11] P.L. Erdős and L. Soukup, Quasi-kernels and quasi-sinks in infinite digraphs, Discrete Math. 309 (2009) 3040-3048.

doi:10.1016/j.disc.2008.08.006

[12] A.S. Fraenkel, Combinatorial game theory foundations applied to digraph kernels, Electron. J. Combin. 4 (1997) \#R10.

[13] H. Galeana-Sánchez and M. Guevara, Some sufficient conditions for the existence of kernels in infinite digraphs, Discrete Math. 309 (2009) 3680-3693. doi:10.1016/j.disc.2008.01.025

[14] H. Galeana-Sánchez and C. Hernández-Cruz, Cyclically k-partite digraphs and kkernels, Discuss. Math. Graph Theory 31 (2011) 63-78. doi:10.7151/dmgt.1530

[15] H. Galeana-Sánchez and C. Hernández-Cruz, $k$-kernels in generalizations of transitive digraphs, Discuss. Math. Graph Theory 31 (2011) 293-312. doi:10.7151/dmgt.1546

[16] H. Galeana-Sánchez and C. Hernández-Cruz, On the existence of $(k, l)$-kernels in digraphs with a given circumference, AKCE Int. J. Graphs Combin. (2013), to appear. 
[17] H. Galeana-Sánchez and C. Hernández-Cruz, k-kernels in k-transitive and k-quasitransitive digraphs, Discrete Math. 312 (2012) 2522-2530.

doi:10.1016/j.disc.2012.05.005

[18] H. Galeana-Sánchez and C. Hernández-Cruz, k-kernels in multipartite tournaments, AKCE Int. J. Graphs Combin. 8 (2011) 181-198.

[19] H. Galeana-Sánchez, C. Hernández-Cruz and M.A. Juárez-Camacho, On the existence and number of $(k+1)$-kings in $k$-quasi-transitive digraphs, Discrete Math. 313 (2013) 2582-2591.

doi:10.1016/j.disc.2013.08.007

[20] H. Galeana-Sánchez and H.A. Rincón, A sufficient condition for the existence of k-kernels in digraphs, Discuss. Math. Graph Theory 18 (1998) 197-204. doi:10.7151/dmgt.1075

[21] A. Ghouila-Houri, Caractérization des graphes non orientés dont onpeut orienter les arrêtes de manière à obtenir le graphe dune relation dordre, Comptes Rendus de l'Académie des Sciences Paris 254 (1962) 1370-1371.

[22] P. Hell and C. Hernández-Cruz, On the complexity of the 3-kernel problem in some classes of digraphs, Discuss. Math. Graph Theory 34 (2014) 167-201. doi:10.7151/dmgt.1727

[23] P. Hell and J. Nešetřil, Graphs and Homomorphisms (Oxford University Press, 2004). doi:10.1093/acprof:oso/9780198528173.001.0001

[24] M. Kucharska and M. Kwaśnik, On $(k, l)$-kernels of special superdigraphs of $P_{m}$ and $C_{m}$, Discuss. Math. Graph Theory 21 (2001) 95-109. doi:10.7151/dmgt.1135

[25] M. Kwaśnik, On $(k, l)$-kernels on graphs and their products, Doctoral Dissertation, Technical University of Wrocław, Wrocław, 1980.

[26] M. Kwaśnik, The generalizaton of Richardson's theorem, Discuss. Math. 4 (1981) $11-14$.

[27] M. Kwaśnik, A. Włoch and I. Włoch, Some remarks about $(k, l)$-kernels in directed and undirected graphs, Discuss. Math. 13 (1993) 29-37.

[28] V. Neumann-Lara, Seminúcleos de una digráfica, Anales del Instituto de Matemáticas II (1971).

[29] M. Richardson, On weakly ordered systems, Bull. Amer. Math. Soc. 52 (1946) 113116. doi:10.1090/S0002-9904-1946-08518-3

[30] R. Rojas-Monroy and I. Villarreal-Valdés, Kernels in infinite digraphs, AKCE Int. J. Graphs Combin. 7 (2010) 103-111.

[31] W. Szumny, A. Włoch and I. Włoch, On $(k, l)$-kernels in D-join of digraphs, Discuss. Math. Graph Theory 27 (2007) 457-470.

doi:10.7151/dmgt.1373 
[32] W. Szumny, A. Włoch and I. Włoch, On the existence and on the number of $(k, l)$ kernels in the lexicographic product of graphs, Discrete Math. 308 (2008) 4616-4624. doi:10.1016/j.disc.2007.08.078

[33] J. von Neumann, O. Morgenstern, Theory of Games and Economic Behavior (Princeton University Press, Princeton, 1953).

Received 8 December 2010

Revised 16 May 2013

Accepted 16 May 2013 